F. Jondral

Nagoya Math. J.

Vol. 81 (1981), 113-122

\title{
SOME REMARKS ABOUT GENERALIZED FUNCTIONALS OF COMPLEX WHITE NOISE
}

\author{
FRIEDRICH JONDRAL
}

\section{Introduction}

Let us start with a short survey concerning complex white noise (for details see Hida [3]): Let $\mathscr{S}$ be the space of real valued rapidly decreasing testing functions and $\mathscr{S}_{c}=\mathscr{S}+i \mathscr{S}=\{\zeta ; \zeta=\xi+i \eta ; \xi, \eta \in \mathscr{S}\}$ its complexification. The dual space $\mathscr{S}_{c}^{*}$ of $\mathscr{S}_{c}$ is the space of tempered distributions. By $\mathfrak{B}$ we denote the $\sigma$-algebra generated by the cylinder sets of $\mathscr{S}_{c}^{*}$. On $\mathscr{S}^{*}$ and $i \mathscr{S}^{*}$ the measures $\mu_{1}$ respectively $\mu_{2}$ are given. Both are measures of real white noise with variances $1 / 2$. On $\mathscr{S}_{c}^{*}$ the product measure $\nu=$ $\mu_{1} \times \mu_{2}$ is introduced. Hida [2] proved the equality

$$
C(\zeta)=\int_{\mathscr{S}_{c}^{*}} e^{i\langle z, \zeta\rangle+\overline{\langle\langle z, \zeta\rangle}} d \nu(z)=e^{-\|\zeta\|^{2}}, \quad \zeta \in \mathscr{S}_{c} .
$$

$\|\cdot\|$ is the $L_{c}^{2}\left(R^{1}\right)$-norm. The form $\langle z, \zeta\rangle=\langle x+i y, \xi+i \eta\rangle$, which connects $\mathscr{S}_{c}^{*}$ and $\mathscr{S}_{c}$ is linear in $z$ and antilinear in $\zeta$.

The measure space $\left(\mathscr{S}_{c}^{*}, \mathfrak{B}, \nu\right)$ is called complex white noise.

Remarks. (1) Every $z \in \mathscr{S}_{c}^{*}$ may be interpreted as a realization of the complex white noise, which is the time derivative of a complex Brownian motion.

(2) If $\zeta \in \mathscr{S}_{c}$ is fixed, $\langle z, \zeta\rangle$ is a random variable on $\left(\mathscr{S}_{c}^{*}, \mathfrak{R}, \nu\right)$.

We are interested in the space $\left(L_{c}^{2}\right)=L_{c}^{2}\left(\mathscr{S}_{c}^{*}, \mathfrak{B}, \nu\right)$ of square integrable functionals of complex white noise.

Let $\left\{\omega_{n} ; \omega_{n} \in \mathscr{S}_{c}\right\}$ be a complete orthonormal system (cons) in $L_{c}^{2}\left(\boldsymbol{R}^{1}\right)$. The subspace $\mathscr{H}_{(m, n)}$ of $\left(L_{c}^{2}\right)$, which is spanned by the family

$$
\left\{\prod_{k} \frac{1}{\sqrt{p_{k} ! q_{k} !}} H_{p_{k}, q_{k}}\left(\left\langle z, w_{j_{k}}\right\rangle,\left\langle\overline{z, \omega_{j_{k}}}\right\rangle\right), \sum_{k} p_{k}=m, \sum_{k} q_{k}=n\right\},
$$

Received July 2, 1979. 
is called complex multiple Wiener integral of order $(m, n)$, where $H_{p_{k}, q_{k}}(z, \bar{z})$ is the complex Hermite polynomial of degree $\left(p_{k}, q_{k}\right)$.

The Wiener-Itô-decomposition of $\left(L_{c}^{2}\right)$ is given by

$$
\left(L_{c}^{2}\right)=\sum_{n=0}^{\infty} \oplus\left\{\sum_{k=0}^{n} \oplus \mathscr{H}_{(k, n-k)}\right\} .
$$

The transformation $\mathscr{T}$, defined by

$$
(\mathscr{T} \varphi)(\zeta)=\int_{\mathscr{S}_{c}^{*}} e^{i\langle z, \zeta\rangle+i \overline{\langle z, \zeta\rangle}} \bar{\varphi}(z) d \nu(z), \zeta \in \mathscr{S}_{c}, \varphi \in\left(L_{c}^{2}\right),
$$

maps $\left(L_{c}^{2}\right)$ onto a reproducing kernel Hilbert space. Moreover, $\mathscr{T}$ is an isomorphism between $\mathscr{H}_{(m, n)}$ and $\hat{L}_{c}^{2}\left(\boldsymbol{R}^{m}\right) \otimes \overline{\hat{L}}_{c}^{2}\left(\boldsymbol{R}^{n}\right)$, which is the space of square integrable functions, symmetric in the first $m$ and in the last $n$ variables.

Another important tool of the white noise analysis is the following theorem, concerning the integral representation of $\varphi \in \mathscr{H}_{(m, n)}$ :

For every $\varphi \in \mathscr{H}_{(m, n)}$ there exists one and only one function $F_{\varphi} \in \hat{L}_{c}^{2}\left(\boldsymbol{R}^{m}\right) \otimes$ $\overline{\hat{L}}_{c}^{2}\left(\boldsymbol{R}^{n}\right)$, which satisfies:

$$
\begin{gathered}
(\mathscr{T} \varphi)(\zeta)=i^{m+n} C(\zeta) \int_{\boldsymbol{R}^{m+n}} \cdots \int F_{\varphi}\left(u_{1}, \cdots, u_{m} ; u_{m+1}, \cdots, u_{m+n}\right) \\
\times \zeta\left(u_{1}\right) \cdots \zeta\left(u_{m}\right) \bar{\zeta}\left(u_{m+1}\right) \cdots \bar{\zeta}\left(u_{m+n}\right) d u_{1} \cdots d u_{m+n}, \\
\|\varphi\|_{\left(L_{c}^{2}\right)}=\sqrt{m ! n !}\left\|F_{\varphi}\right\|_{L\left(\boldsymbol{R}^{m+n}\right)}
\end{gathered}
$$

\section{Generalized complex Brownian functionals}

Let $\mathfrak{P}_{n}=\left\{\Delta_{k}\right\}$ be an uniform partition of $\boldsymbol{R}^{1}$. Formally $\{(d B(t)) / \sqrt{d t}\}$, the projective system of $\left\{\left(\Delta_{k} B\right) / \sqrt{\left|\Delta_{k}\right|}, \Delta_{k} \in \mathfrak{P}_{n}\right\}$, defines an equally dense cons in $\mathscr{H}_{(1,0)}$.

Remark. For the notion of equally dense cons see P. Lévy [6], chapitre III.

Starting from the coordinate system in $\mathscr{H}_{(1,0)}$, we define coordinate systems in every $\mathscr{H}_{(m, n)}$ by using complex Hermite polynomials with parameter, which are given by

$$
H_{p, q}^{*}\left(z, \bar{z} ; \sigma^{2}\right)=\frac{\left(-\sigma^{2}\right)^{p+q}}{p ! q !} e^{z \bar{z} / \sigma^{2}} \frac{\partial^{p+q}}{\partial \bar{z}^{p} \partial z^{q}} e^{-z \bar{z} / \sigma^{2}}, \quad \sigma^{2}>0 ; p, q \geqq 0 .
$$

As coordinate systems in $\mathscr{H}_{(m, n)}$ we get 


$$
\begin{gathered}
\prod_{j=1}^{p}\left\{H_{1,0}^{*}\left(B^{\cdot}\left(t_{j}\right), \bar{B}^{\cdot}\left(t_{j}\right) ; \frac{1}{d t_{j}}\right) \sqrt{d t_{j}}\right\} \prod_{j=p+1}^{p+q}\left\{H_{0,1}^{*}\left(B^{\cdot}\left(t_{j}\right), \bar{B}^{\cdot}\left(t_{j}\right) ; \frac{1}{d t_{j}}\right) \sqrt{d t_{j}}\right\}, \\
\prod_{j}\left\{\frac{1}{\sqrt{p_{j} ! q_{j} !}} H_{p_{j}, q_{j}}^{*}\left(B^{*}\left(t_{j}\right), \bar{B}^{\cdot}\left(t_{j}\right) ; \frac{1}{d t_{j}}\right)\left[\sqrt{d t_{j}}\right]^{p_{j}+q_{j}}\right\}
\end{gathered}
$$

(all $t_{j}$ 's are different), $\sum_{j} p_{j}=p, \sum_{j} q_{j}=q$.

Remark. (2.2) and (2.3) are not to be considered as actual coordinate systems. They are only formal expressions, but, just as in the case of real white noise, they are consistent with the partitions $\mathfrak{P}_{n}$ by an addition formula:

$$
\begin{aligned}
& H_{p, q}^{*}\left(z_{1}+z_{2}, \overline{z_{1}+z_{2}} ; \sigma_{1}^{2}+\sigma_{2}^{2}\right) \\
& \quad=\sum_{k=0}^{p} \sum_{j=0}^{q} H_{p-k, q-j}^{*}\left(z_{1}, \bar{z}_{1} ; \sigma_{1}^{2}\right) H_{k, j}^{*}\left(z_{2}, \bar{z}_{2} ; \sigma_{2}^{2}\right) .
\end{aligned}
$$

Formula (1.4) tells us, that the integral representations of $\mathscr{H}_{(m, 0)}$ functionals are regular functionals in P. Lévy's sense. We are going to generalize the $\mathscr{H}_{(m, n)}$-functionals in such a manner, that also normal functionals in the sense of P. Lévy may be integral representations of complex Brownian functionals. The following example suggests, how to define generalized functionals of complex white noise:

ExAmple 1. Let a version of complex Brownian motion be given by

$$
B(t, z)=\left\langle z, \chi_{[t \wedge 0, t \vee 0]}\right\rangle
$$

and $\left\{3_{k}\right\}, 8_{k}=\left\{\Delta_{k_{j}}\right\}$, be a sequence of partitions of $\boldsymbol{R}^{1}$ into disjoint intervals

$$
\Delta_{k_{j}} B(t, z)=B\left(t_{k_{j+1}}, z\right)-B\left(t_{k_{j}}, z\right)=\left\langle z, \chi_{\Delta_{k_{j}}}\right\rangle \text {. }
$$

The functional

$$
\varphi_{k}(z)=\sum_{k_{j}} \frac{1}{\sqrt{2}} a_{k_{j}}\left[\frac{\left\langle z, \chi_{\Delta_{k j}}\right\rangle}{\left|\Delta_{k_{j}}\right|}\right]^{2}\left|\Delta_{k_{j}}\right|, a_{k_{j}} \in C
$$

belongs to $\mathscr{H}_{(2,0)}$. Applying $\mathscr{T}$, we get

$$
\left(\mathscr{T} \varphi_{k}\right)(\zeta)=i^{2} C(\zeta) \sum_{k_{j}} \frac{1}{\sqrt{2}} \bar{a}_{k_{j}} \iint \zeta(u) \zeta(v) \frac{\chi_{\Delta_{k_{j}}}(u) \chi_{\iota_{k_{j}}}(v)}{\left|\Delta_{k_{j}}\right|^{2}} d u d v\left|\Delta_{k_{j}}\right|
$$

We now assume

$$
\sum_{k_{j}} \bar{a}_{k_{j}} \chi_{\Delta_{k_{j}}} \stackrel{(k \rightarrow \infty)}{\longrightarrow} f(u) \in L_{c}^{1}\left(\boldsymbol{R}^{1}\right)
$$


Then we suppose $3_{k}$ to be so fine, that the double integral in (2.7) may be replaced by a Riemannian double sum. We find

$$
\left(\mathscr{T} \varphi_{k}\right)(\zeta)=i^{2} C(\zeta) \frac{1}{\sqrt{2}} \sum_{k_{j}} \bar{a}_{k_{j}} \chi_{\Delta_{k_{j}}}\left(u_{k_{j}}\right) \zeta^{2}\left(u_{k_{j}}\right)\left|\Delta_{k_{j}}\right|
$$

Now, since $k$ increases, $3_{k}$ becomes finer and finer, which finally implies

$$
\begin{aligned}
& \left(\mathscr{T} \varphi_{k}\right)(\zeta) \stackrel{(k \rightarrow \infty)}{\longrightarrow} i^{2} C(\zeta) \frac{1}{\sqrt{2}} \int f(u) \zeta^{2}(u) d u \\
& \quad=i^{2} C(\zeta) \frac{1}{\sqrt{2}} \iint f\left(\frac{u+v}{2}\right) \delta(u-v) \zeta(u) \zeta(v) d u d v
\end{aligned}
$$

From (1.4) we know, that $i^{2} C(\zeta)(1 / \sqrt{2}) \int f(u) \zeta^{2}(u) d u$ is not the image of an $\mathscr{H}_{(2,0)}$-functional under $\mathscr{T}$. Because $\mathscr{T}$ is a one-to-one transformation, we now consider a limit procedure, similarly to the procedure described before, in the space $\mathscr{H}_{(2,0)}$. We see, that a sequence of $\mathscr{H}_{(2,0)}$-functionals converges to a limit, which may not be found in $\mathscr{H}_{(2,0)}$. However, this limit is uniquely determined by the sequence of $\mathscr{H}_{(2,0)}$-functionals. Formally the limit may be written as

$$
\frac{1}{\sqrt{2}} \int f(u)\left[\frac{d B(u)}{d u}\right]^{2} d u
$$

It can be proved, that this limit is an element of $\mathscr{H}_{(2,0)}^{-(2)}$ (for definition see below).

Now we are able to give a definition of generalized complex Brownian functionals:

The space

$$
S^{m}\left(\boldsymbol{R}^{n}\right)=\left\{v ; v \in \mathscr{S}_{c}^{*}\left(\boldsymbol{R}^{n}\right),\left(1+|x|^{2}\right)^{m / 2} \widetilde{F}(v(t)) \in L_{c}^{2}\left(\boldsymbol{R}^{n}\right)\right\},
$$

where $\mathfrak{f}$ denotes the Fourier transform, is called the Sobolev space of (fractional) order $m$ over $\boldsymbol{R}^{n}$ (Lions/Magenes [7]). Provided with the norm

$$
\|\| v\left\|_{S^{m}\left(\boldsymbol{R}^{n}\right)}=\right\|\left(1+|x|^{2}\right)^{m / 2} \widetilde{\mho}(v(t)) \|_{L_{\mathrm{c}}^{2}\left(\boldsymbol{R}^{n}\right)}
$$

$\boldsymbol{S}^{m}\left(\boldsymbol{R}^{n}\right)$ becomes a Hilbert space. We are interested in the symmetric Sobolev space, which is defined by

$$
\hat{\boldsymbol{S}}^{(m+1) / 2,(n+1) / 2}\left(\boldsymbol{R}^{m+n}\right)=\left[\boldsymbol{S}^{(m+1) / 2}\left(\boldsymbol{R}^{m}\right) \otimes S^{(n+1) / 2}\left(\boldsymbol{R}^{n}\right)\right] \cap\left[\hat{L}_{c}^{2}\left(\boldsymbol{R}^{m}\right) \otimes \overline{\hat{L}}_{c}^{2}\left(\boldsymbol{R}^{n}\right)\right] .
$$

Definition 2.1 .

$$
\mathscr{H}_{(m, n)}^{(m+n)}:=\left\{\varphi ; F_{\varphi} \in \hat{S}^{(m+1) / 2,(n+1) / 2}\left(\boldsymbol{R}^{m+n}\right)\right\} .
$$


On $\mathscr{H}_{(m, n)}^{(m+n)}$ we introduce the norm

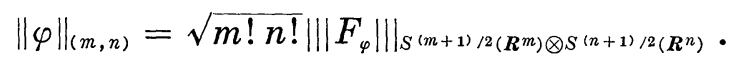

Now $\mathscr{H}_{(m, n)}^{(m+n)}$ is a Hilbert space and the injection $\mathscr{H}_{(m, n)}^{(m+n)} \subset \mathscr{H}_{(m, n)}$ is continuous. $\mathscr{H}_{(m, n)}^{-(m+n)}$, the dual space of $\mathscr{H}_{(m, n)}^{(m+n)}$ is defined in the following manner:

To every $G \in \hat{S}^{-(m+1) / 2,-(n+1) / 2}\left(\boldsymbol{R}^{m+n}\right)$, which is the dual space of $\hat{S}^{(m+1) / 2,(n+1) / 2}\left(\boldsymbol{R}^{m+n}\right)$, we adjoin a functional $\varphi_{G}$ by using $\mathscr{T}^{-1}$ in such a way, that

$$
\left\{\varphi_{G}, \varphi_{F}\right\}_{(m, n)}=m ! n ![G, F]_{(m, n)}
$$

holds for every $\varphi_{F} \in \mathscr{H}_{(m, n)}^{(m+n)}$. The function $F$ is the kernel of the integral representation of $\varphi_{F} \cdot\{\cdot, \cdot\}$ respectively $[\cdot, \cdot]$ are the forms, which connect $\mathscr{H}_{(m, n)}^{-(m+n)}$ and $\mathscr{H}_{(m, n)}^{(m+n)}$ respectively $\hat{\boldsymbol{S}}^{-(m+1) / 2,-(n+1) / 2}\left(\boldsymbol{R}^{m+n}\right)$ and $\hat{\boldsymbol{S}}^{(m+1) / 2,(n+1) / 2}\left(\boldsymbol{R}^{m+n}\right)$.

Definition 2.2. Every $\varphi \in \mathscr{H}_{(m, n)}^{-(m+n)}$ is called a generalized complex Brownian functional of degree $(m, n)$.

EXAMPLE 1 (continued). It is now easy to show, that the Fourier transform of the kernel $f((u+v) / 2) \delta(u-v)$, which appears in (2.10) is a member of $\hat{S}^{-3 / 2}\left(\boldsymbol{R}^{2}\right)$. Consequently the functional (2.11) may be found in $\mathscr{H}_{(2,0)}^{-(2,0)}$.

Finally in this section, we present the relations between the spaces, which were considered: Here $G$ means continuous injection, while $\leftrightarrow$ denotes one-to-one correspondence between two spaces:

Picture 1

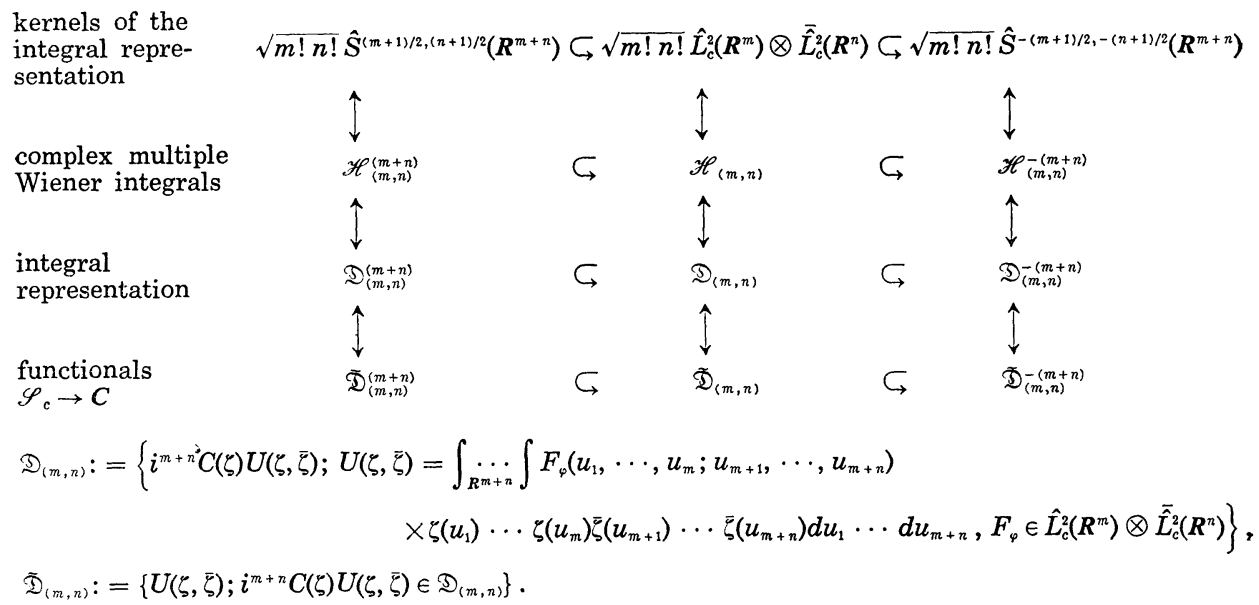


In the next sections we restrict our attention to the complex multiple Wiener integral $\mathscr{H}_{(m, 0)}$, i.e. $n=0$.

\section{Generalized complex random measures}

With the results of section 2 in mind and with the assumption $f\left(u_{1}, \cdots, u_{\ell}\right) \in L_{c}^{1}\left(R^{\ell}\right)$, it is quite easy to show, that the functional

$$
\varphi(z)=\int \underset{\boldsymbol{R} \ell}{\ldots} \int f\left(u_{1}, \cdots, u_{\ell}\right) \prod_{j=1}^{\ell}\left\{H_{m_{j}, 0}^{*}\left(B^{\cdot}\left(u_{j}\right), \bar{B}^{\cdot}\left(u_{j}\right) ; \frac{1}{d u_{j}}\right) d u_{j}\right\},
$$

$u_{j}$ different for $j=1, \cdots, \ell ; \sum_{j} m_{j}=m$, belongs to $\mathscr{H}_{(m, 0)}^{-(m)}$.

The product

$$
\prod_{j=1}^{\ell} \cdot M_{m_{j}}\left(d u_{j}\right):=\left\{\begin{array}{l}
\prod_{j=1}^{\ell}\left\{H_{m_{j}, 0}^{*}\left(B^{\cdot}\left(u_{j}\right), \bar{B}^{\cdot}\left(u_{j}\right) ; \frac{1}{d u_{j}}\right) d u_{j}\right\}, u_{j} \neq u_{k}, \quad \text { if } j \neq k \\
0 \quad \text { otherwise }
\end{array}\right.
$$

$\sum_{j} m_{j}=m$, is a complex random measure. Integrals with respect to this measure are members of $\mathscr{H}_{(m, 0)}^{-(m)}$ and it follows, that the kernel of

$$
\mathscr{T}\left\{\prod_{j=1}^{\ell} \cdot M_{m_{j}}\left(d u_{j}\right)\right\} \text { is } \frac{1}{m !} \prod_{j=1}^{\ell}\left\{\delta_{u}^{m_{j}} d u_{1} \cdots d u_{m_{j}}\right\}
$$

where the measure $\delta_{u}^{m} d u_{1} \cdots d u_{m}$ is given by

$$
\int \underset{\boldsymbol{R}^{m}}{\ldots} \int f\left(u_{1}, \cdots, u_{m}\right) \delta_{u}^{m} d u_{1} \cdots d u_{m}=\int_{\boldsymbol{R}^{1}} f(u, \cdots, u) d u .
$$

Remarks (for the case of real white noise see Hida [3]):

(1) Integrals with respect to generalized complex random measures are not integrals in the usual sense, but they are continuous analogues of polynomials.

(2) Integrals with respect to $\prod_{j=1}^{m} \cdot M_{j}\left(d u_{j}\right)$ are members of, $\mathscr{H}_{(m, 0)}$, the complex multiple Wiener integral of degree $(m, 0)$.

(3) There exist elements of $\mathscr{H}_{(m, 0)}^{-(m)}$ which may not be represented as integrals with respect to a generalized complex random measure.

EXAmple 2. If we choose a function $\eta$ from $\mathscr{S}_{c}$, we are able to show, that the generalized stochastic process

$$
X(\eta)=\int \hat{\eta}(u) M_{m}(d u)
$$


lives in $\mathscr{H}_{(m, 0)}^{-(m)} . \quad \hat{\eta}(u)=\widetilde{\Im}(\eta(t))$ is the Fourier transform of $\eta(t)$. Applying $\mathscr{T}$ we get

$$
\begin{aligned}
(\mathscr{T} X(\eta))(\zeta) & =\frac{i^{m} C(\zeta)}{m !} \int_{-\infty}^{\infty} \hat{\eta}(u) \zeta^{m}(u) d u \\
& =\frac{i^{m} C(\zeta)}{m !} \int_{\boldsymbol{R}^{m}} \int \hat{\eta}\left[\frac{u_{1}+u_{2}+\cdots+u_{m}}{m}\right] \zeta\left(u_{1}\right) \zeta\left(u_{2}\right) \cdots \zeta\left(u_{m}\right) \delta_{u}^{m} d u_{1} \cdots d u_{m}
\end{aligned}
$$

and the kernel of the integral representation of $X(\eta)$ turns out to be

$$
F_{X(\eta)}=\hat{\eta}\left[\frac{u_{1}+\cdots+u_{m}}{m}\right] \delta_{u}^{m} .
$$

Now we have to prove $F_{X(\eta)} \in \hat{S}^{-(m+1) / 2}$ : Take $\psi \in \mathscr{S}_{c}\left(\boldsymbol{R}^{m}\right)$, then

$$
\begin{aligned}
& \left(F_{X(\eta)}, \widetilde{\mho}(\psi)\right) \\
& =(2 \pi)^{-m / 2} \int \underset{R^{2 m}}{\ldots} \overline{\hat{\eta}\left[\frac{u_{1}+\cdots+u_{m}}{m}\right] \delta_{u}^{m}} e^{-i \sum_{j=1}^{m} u_{j} t_{j}} \psi\left(t_{1}, \cdots, t_{m}\right) \\
& \times d t_{1} \cdots d t_{m} d u_{1} \cdots d u_{m}
\end{aligned}
$$

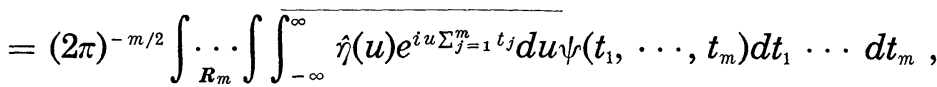

$$
\begin{aligned}
& \mathfrak{\zeta}\left(F_{X(\eta)}\right)=(2 \pi)^{-m / 2} \int_{-8}^{\infty} \hat{\eta}(u) e^{i u \sum_{j=1}^{m} t_{j}} d u \\
& \times\left\|\frac{1}{\left[1+\sum_{j=1}^{m} t_{j}^{2}\right]^{(m+1) / 4}} \mathfrak{F}\left(F_{X(\eta)}\right)\right\|^{2} \\
& =(2 \pi)^{-m} \int \underset{\boldsymbol{R}_{m}}{\ldots} \int \frac{\left|\int_{-\infty}^{\infty} \hat{\eta}(u) e^{i u \Sigma_{j=1}^{m} t_{j}} d u\right|^{2}}{\left(1+\sum_{j=1}^{m} t_{j}^{2}\right)^{(m+1) / 2}} d t_{1} \cdots d t_{m}<\infty .
\end{aligned}
$$

ExAmple 3. Take $F \in L_{c}^{2}\left(R^{1}\right)$, then the stochastic process

$$
Y(t)=\int^{t} F(t-u) M_{m}(d u)
$$

lives in $\mathscr{H}_{(m, 0)}^{-(m)}$ and it turns out to be a stationary process. The shift of $t$ comes from the flow of the Brownian motion.

\section{Analytic functionals of complex white noise in the sense of Gâteaux}

Instead of the space $\mathscr{H}_{(m, 0)}^{-(m)}$ we may-because of isomorphism-consider the space $\tilde{D}_{(m, 0)}^{-(m)}$, which is a space of functionals with domain $\mathscr{S}_{\mathrm{c}}$ and with values in $C$. This remark enables us to refer to the theory of Gâteaux [1] and Taylor [8], which must be modified only a little bit: 
Definition 4.1 (Taylor). Let $D$ be an open subset of $\mathscr{S}_{\mathrm{c}}$ and $U(\zeta)$ a functional defined on $D$. $U(\zeta)$ is called analytic in $D$, if it is continuous in $D$ and admits a Gâteaux variation at every point of $D . U(\zeta)$ is called analytic at $\zeta_{0}$, if it is analytic in a neighbourhood of $\zeta_{0}$.

By $\mathscr{S}^{+}$we denote the space $\sum_{m=0}^{\infty} \oplus \mathscr{H}_{(m, 0)}^{(m)}$. A linear continuous functional on $\mathfrak{S}^{+}$must be a linear combination of members of the $\mathscr{H}_{(m, 0)}^{-(m)}$ 's, $m=0,1,2, \cdots$ :

$$
\varphi=\sum_{m=0}^{\infty} \varphi_{m} \in \mathfrak{S}^{-}, \varphi_{m} \in \mathscr{H}_{(m, 0)}^{-(m)} .
$$

Take $\psi=\sum_{m=0}^{\infty} \psi_{m} \in \mathfrak{S}^{+}$, then the form $] \cdot, \cdot\left[\right.$ connecting $\mathfrak{S}_{\mathcal{C}}^{-}$and $\mathfrak{S}^{+}$is given by

$$
] \varphi, \psi\left[:=\sum_{m=0}^{\infty}\left\{\varphi_{m}, \psi_{m}\right\}_{(m, 0)} .\right.
$$

Now we got the space tripel

$$
\mathfrak{S E}^{+} \subset \mathfrak{H C}_{\mathcal{C}} \subset \mathfrak{S}^{-}, \quad \mathfrak{S}_{\mathcal{E}}=\sum_{m=0}^{\infty} \oplus \mathscr{H}_{(m, 0)} .
$$

Definition 4.2. $\varphi=\sum_{m=0}^{\infty} \varphi_{m} \in \mathfrak{S}^{-}$is called an analytic functional of complex white noise in the sense of Gâteaux, if a sequence $\left\{i^{m} C(\zeta) U_{m}(\zeta)\right.$; $m=0,1,2, \cdots\}$ of integral representations is adjuncted to the sequence $\left\{\varphi_{m} ; m=0,1,2, \cdots\right\}$, such that $\sum_{m=0}^{\infty} U_{m}(\zeta)$ is an analytic functional in $\mathscr{S}_{c}$.

EXAMPLE 4. $\varphi(z)=e^{i\langle z, \zeta\rangle}, \zeta \in \mathscr{S}_{c}$, is an analytic functional of complex white noise in the sense of Gâteaux.

Remark. Note, that the functional $\varphi(z)=e^{i\langle z, \zeta\rangle}$ is an element of $\mathfrak{S E}^{-}$. In the case of real white noise $e^{i\langle x, \xi\rangle}, \xi \in \mathscr{S}$, may not be found in $\mathfrak{S}_{\text {real }}^{-}$, but with a normalization we have $e^{i\langle x, \xi\rangle+1 /(2 d t)} \in \mathfrak{F}_{\text {real }}^{-}$.

\section{Differential calculus for functionals of complex white noise}

With every $\varphi \in \mathscr{H}_{(m, 0)}^{(m)}$ is associated its integral representation

$$
\begin{aligned}
(\mathscr{T} \varphi)(\zeta) & =i^{m} C(\zeta) \int_{\boldsymbol{R}^{m}} \ldots \int F_{\varphi}\left(u_{1}, \cdots, u_{m}\right) \zeta\left(u_{1}\right) \cdots \zeta\left(u_{m}\right) d u_{1} \cdots d u_{m} \\
& =i^{m} C(\zeta) U(\zeta) .
\end{aligned}
$$

We compute the functional derivative of $U(\zeta)$ in P. Lévy's sense and get

$$
U_{\zeta}^{\prime}(t ; \zeta)=m \int_{R^{m-1}}^{\ldots} \int F_{\varphi}\left(t ; u_{2}, \cdots, u_{m}\right) \zeta\left(u_{2}\right) \cdots \zeta\left(u_{m}\right) d u_{2} \cdots d u_{m}
$$


If $t$ is fixed, we can associate a new complex Brownian functional with $U_{\zeta}^{\prime}(t ; \zeta)$ :

$$
Z(t ; z)=\mathscr{T}^{-1}\left(i^{m-1} C(\zeta) U_{\zeta}^{\prime}(t ; \zeta)\right) \in \mathscr{H}_{(m-1,0)}^{(m-1)}
$$

$Z(t ; z)$ is then called the $d / d z(t)$-derivative of $\varphi(z)$.

Remarks. (1) The system $\left\{Z(t ; z) ; t \in T \subset R^{1}\right\}$ may be interpreted as a stochastic process with values in $\mathscr{H}_{(m-1,0)}^{(m-1)}$.

(2) If we interpret $\varphi(z, \bar{z})=\varphi(x+i y, x-i y)=\varphi(x, y)$ as a functional of the two variables $x, y \in \mathscr{S}^{*}$, we may define the $\partial / \partial x(t)$ - and $\partial / \partial y(t)-$ derivatives of $\varphi$ by the help of $U_{\xi}^{\prime}(t ; \xi, \eta)$ and $U_{\eta}^{\prime}(t ; \xi, \eta)$. Also higher order derivatives may be defined via the higher order functional derivatives of $U(\zeta)$ in the sense of P. Lévy.

Example 5. P. Lévy's stochastic area may be written in the form

$$
S(T)=\frac{1}{2 i} \int_{0}^{T}\{\bar{B}(s) d B(s)-B(s) d \bar{B}(s)\}
$$

where $B(t)$ is a version of the Brownian motion. $S(1)$ is a member of $\mathscr{H}_{(1,1)}$ (Hida [2]).

The kernel of the integral representation of $S(1)$ turns out to be

$$
F(u, v)= \begin{cases}\frac{1}{2 i} & \text { if } 0 \leqq v<u \leqq 1 \\ \frac{-1}{2 i} & \text { if } 0 \leqq u<v \leqq 1 \\ 0 & \text { elsewhere }\end{cases}
$$

Picture 2

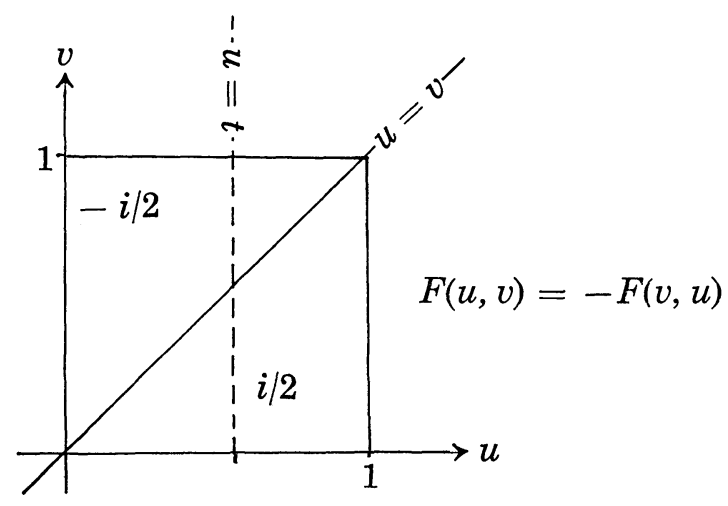


We consider the integral representation

$$
\begin{aligned}
(\mathscr{T} S(1))(\zeta) & =i^{2} C(\zeta) \iint F(u, v) \zeta(u) \bar{\zeta}(v) d u d v \\
& =i^{2} C(\zeta) U(\xi, \eta)
\end{aligned}
$$

and compute

$$
U_{\xi}^{\prime}(t ; \xi, \eta)=\int F(t ; v)\{\bar{\zeta}(v)-\zeta(v)\} d v
$$

From (5.6) it is easy to conclude, that, if $t \in[0,1]$

$$
\frac{\partial S(1 ; x, y)}{\partial x(t)}=i \bar{B}(t, z)-\frac{i}{2} \bar{B}(1, z)-i B(t, z)+\frac{i}{2} B(1, z) \in \mathscr{H}_{(1,0)} \oplus \mathscr{H}_{(0,1)} .
$$

Acknowledgement. The author expresses his thanks to Professor Dr. Takeyuki Hida (Nagoya University, Japan) and to Professor Dr. Ernst Henze (Technische Universität Braunschweig, Germany) for their suggestions and for many discussions.

\section{REFERENCES}

[1] Gâteaux, R., Sur diverses questions de calcul fonctionel, Bullétin de la Société Math. de France, vol. 50 (1922), pp. 1-21.

[2] Hida, T., Complex white noise and infinite dimensional unitary group, lecture note, Nagoya University (1971).

[3] - Brownian motion, Iwanami Publ. Comp., Tokyo (1975) (in Japanese, an English translation will appear in Springer-Verlag).

[4] - Analysis of Brownian functionals, lecture note (2nd edition), Carleton University (Ottawa) (1978).

[5] - Generalized multiple Wiener integrals, Proceedings of the Japan Academie, vol. 54, ser. A, no. 3 (1978), pp. 55-58.

[ 6 ] Lévy, P., Problèmes concrets d'analyse fonctionelle, Gauthier-Villars, Paris (1951).

[7] Lions, J. L. and E. Magenes, Non-homogeneous boundary value problems and applications (vol. 1), Springer-Verlag, Berlin-Heidelberg-New York (1972).

[8] Taylor, A. E., Analytic functions in general analysis, Annali della R. Scoula Normale Superiore de Pisa (2), vol. 6 (1937), pp. 277-292.

Institut für Angewandte Mathematik

Technische Universität Braunschweig

Current address:

$A E G-T E L E F U N K E N$,

Ulm (Donau) 